\title{
1 Mosquito excreta reveals circulation of West Nile virus and its underlying ecosystem.
}

2

3 Grégory L'Ambert ${ }^{1}$, Mathieu Gendrot ${ }^{2,3,4}$, Sébastien Briolant ${ }^{2,3,4}$, Agnès Nguyen ${ }^{5}$, Sylvain

4 Pages ${ }^{1}$, Laurent Bosio ${ }^{6,7}$, Vincent Palomo ${ }^{6,7}$, Nicolas Gomez ${ }^{2,3,4}$, Nicolas Benoit ${ }^{2,3,4}$, Hélène

5 Savini ${ }^{3,8}$, Bruno Pradines ${ }^{2,3,4,9}$, Guillaume André Durand ${ }^{6,7}$, Isabelle Leparc-Goffart ${ }^{6,7}$, Gilda

6 Grard $^{6,7}$, Albin Fontaine $2,3,4^{*}$

7

$8{ }^{1}$ Entente interdépartementale pour la démoustication du littoral méditerranéen (EID

9 Méditerranée), Montpellier, France.

$10{ }^{2}$ Unité Parasitologie et Entomologie, Département Microbiologie et maladies infectieuses,

11 Institut de Recherche Biomédicale des Armées (IRBA), Marseille, France.

$12{ }^{3}$ Aix Marseille Univ, IRD, SSA, AP-HM, VITROME, Marseille, France.

$13{ }^{4}$ IHU Méditerranée Infection, Marseille, France.

$14{ }^{5}$ Microsynth France, 170 avenue Gabriel Péri 69120 Vaulx-en-Velin, France.

$15{ }^{6}$ Unité des Virus Émergents (UVE : Aix-Marseille Univ-IRD 190-Inserm 1207), Marseille,

16 France.

$17{ }^{7}$ Institut de Recherche Biomédicale des Armées, Centre National de Référence des Arbovirus,

18 Marseille, France.

$19{ }^{8}$ Service des Maladies Infectieuses, Hôpital d'Instruction des Armées Laveran, Marseille,

20 France.

$21{ }^{9}$ Centre National de Référence du Paludisme, Marseille, France. 


\section{Abstract}

25 Emerging and endemic mosquito-borne viruses can be difficult to detect and monitor because

26 they often cause asymptomatic infections in human or vertebrate animals or cause nonspecific

27 febrile illness with a short recovery waiting period. Cases' detection in vertebrate hosts can be complemented by entomological surveillance, but this method is not adapted to low infection

29 rates in mosquito populations that typically occur in low or non-endemic areas. We identified

30 West Nile Virus circulation in Camargue, a wetland area in South of France, using a cost

31 effective innovative xenomonitoring method based on the molecular detection of virus in

32 excreta from trapped mosquitoes. We also succeeded at identifying the mosquito community

33 diversity dynamic on several sampling sites, together with the vertebrate hosts on which they

34 fed prior to be captured using amplicon-based metagenomic on mosquito excreta without

35 processing any mosquito. Mosquito excreta-based virus surveillance can be considered as a

36 cost-effective and non-invasive strategy that offers the additional asset to reveal the ecological

37 network underlying arbovirus circulation. 


\section{Main}

West Nile viruses (WNVs) are mosquito-borne flaviviruses (from the Flaviviridae family, Flavivirus genus and belonging to the Japanese encephalitis (JE) serogroup) that circulate through several lineages into complex transmission cycles involving mosquitoes from the Culex genus and several vertebrate host species, with wild birds as amplifying hosts $^{1,2}$. WNVs can cause severe and potentially fatal neurological disease in vertebrate hosts, including birds, Equidae and humans ${ }^{3}$. Native to Africa, WNVs from lineage 1 were first introduced in Europe presumably through migratory birds in the $1960 \mathrm{~s}^{4}$ where they caused mainly occasional self-limited outbreaks and sporadic cases ${ }^{5}$ essentially restricted to rural areas. Major European outbreaks occurred in Camargue in $1962-1963^{6}$ and Romania in $1996^{7}$.

The emergence of lineage 2 in southeastern Hungary in 2004 was associated with a significant upsurge of human and animal cases in several European countries. The largest outbreak was recorded in 2018 with 11 countries reporting 1,548 locally WNV infections, a number that exceeded the cumulative number of all reported infections between 2010 and $2017^{8}$. WNV lineage 1 and 2 are now circulating in endemic cycles in Europe ${ }^{5}$, causing disease incidence in human every year that tend to be increasingly associated to more urbanized environment.

Ecological interactions among bird species that led to WNVs introduction, amplification, and maintenance in local reservoirs, a prerequisite before the virus emergence in humans, are not yet fully understood.

Standard entomological surveillance provides the opportunity to monitor arboviruses circulation in their enzootic cycles based on the processing of thousands of mosquitoes that is cost prohibitive and time consuming. This surveillance method also poses logistical constraints and is thus not adapted to low infection rates in mosquito populations that typically occur in low or non-endemic areas. It has been demonstrated that mosquitoes infected with different arboviruses can excrete large amount of virus genomic RNA ${ }^{9-11}$. Here, 
64 we implemented an innovative entomological surveillance strategy inspired from

65 environmental DNA (eDNA) sequencing and molecular xenomonitoring (MX) ${ }^{12}$ that

66 drastically decrease drawbacks associated with standard entomological surveys while

67 providing additional benefits by screening excreta from trapped mosquitoes. We developed a

68 3D printed lodging that can be adapted on main standard mosquito traps, which offer shelter,

69 food, and a toilet room for trapped mosquitoes. Our xenomonitoring strategy succeeded at

70 detecting two WNV in excreta from the mosquito fauna sampled over a two-month

71 longitudinal study in summer 2020 in Camargue, a wetland area (Rhône delta) in South of

72 France. One of these viruses phylogenetically grouped with a virus from the lineage 1 isolated

73 in 2015 in a horse in the same region. Trapped mosquito excreta were also collected in Mali.

74 Using a single amplicon-based metagenomic approach, we succeeded to monitor the mosquito

75 fauna diversity across different sampling sites and times without processing any mosquitoes.

76 Importantly, we also succeed at identifying vertebrate hosts on which mosquitoes fed prior to

77 be captured. Altogether, we showed that the use of mosquito excreta is efficient to trace

78 arbovirus circulation at low cost and can give precious clues to characterize the ecosystem

79 underlying arbovirus emergence.

$81 \quad$ Results

$82 \quad$ Virus screening in mosquito excreta.

83 A total of 86 excreta samples were analyzed in this study ( 80 excreta samples from

84 Camargue, France and 6 from Gao, Mali). These excreta were collected at the end of summer

852020 from trapped mosquitoes contained into the 3D printed MX adapter that has been

86 specifically designed to increase trapped mosquitoes' survival and to facilitate the recovery of

87 their excreta over several days (Figure 1). A total of 6,845 mosquitoes were recovered from

88 traps in Camargue, comprising 2,819 Culex spp., 3,922 Ochlerotatus/Aedes spp., 91 
Anopheles spp. and 13 Culiseta spp. mosquitoes (Supplementary file 1). Anopheles spp. were morphologically identified as An. hyrcanus sensu lato and as species from the An.

maculipennis complex (which includes An. atroparvus). Specimens not belonging to the

Culicidae family were also collected in traps but were not considered in this study. Trapped

fauna from Mali were not brought back to Marseille laboratory for analysis. WNV genome

was detected by one-step reverse transcription quantitative polymerase chain reaction (RT-

qPCR) on excreta samples from 2 traps $(2.3 \%)$ in Camargue, collected on location $F$ the $15^{\text {th }}$ of September 2020 (cycle threshold (Ct) of 31) and on location G the $22^{\text {nd }}$ of September 2020 (cycle threshold (Ct) of 33) (Figure 2). No excreta samples were found positive for WNV in Mali, nor Usutu virus (USUV) in both Camargue and Mali. Excreta samples from Mali were also tested negative for Plasmodium .spp., the causative agents of malaria, in Mali.

A

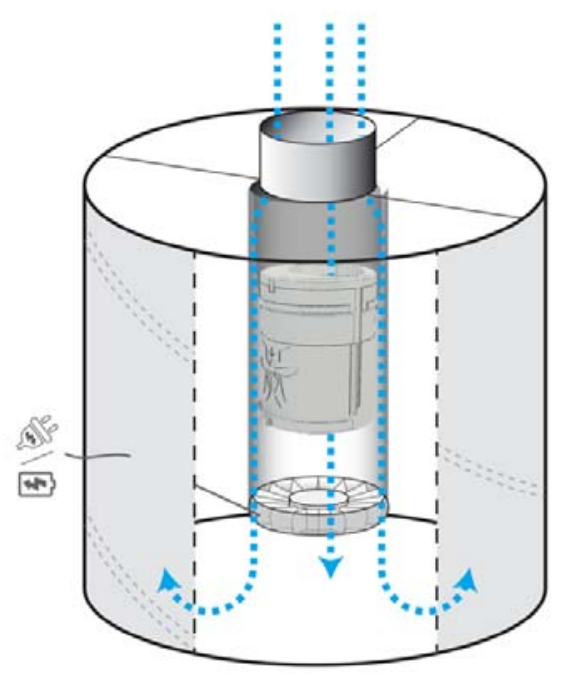

B

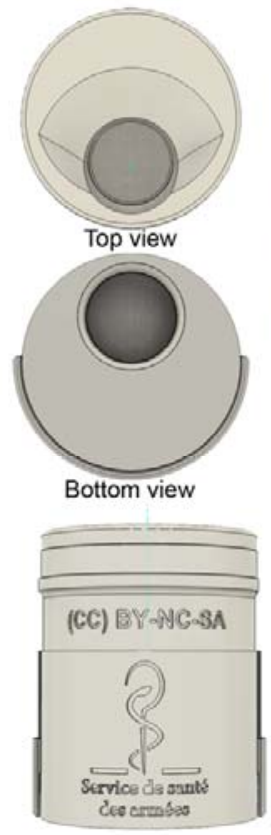

Rear view
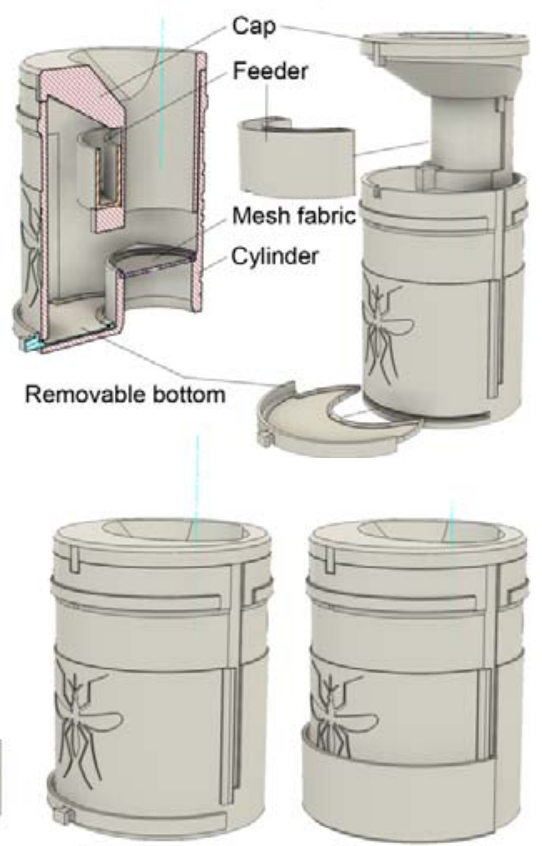

Catching mode

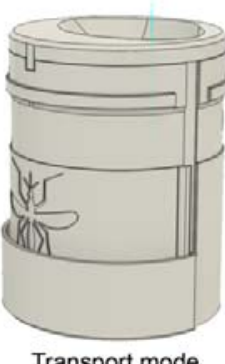

Transport mode

Figure 1: 3D representations of the $M X$ adapter designed to increase trapped mosquitoes' longevity and to collect their excreta for a virus surveillance purpose. (A)

104 Schematic of the MX adapter inside the BG sentinel trap (BGS) in catching mode. (B) 
105 Different views of the adapter MX. All components are visible in the cross-sectional and

106 exploded views of the MX adapter: the cap, the mosquito feeder, the ring covered with mesh

107 fabric, the cylinder, and the removable bottom on which can be placed a filter paper to collect

108 mosquito excreta. In transport mode, the sliding gate covers the open slot in absence of the

109 removable bottom. The MX adapter was created on Fusion 360 (AutoDesk). MX adapter is

110 under the Creative Commons (CC) license BY-NC-SA (Licensees may copy, distribute,

111 display and make derivatives only for non-commercial purposes and by giving credits to the

112 authors). All printable files are provided in Supplementary file 3.
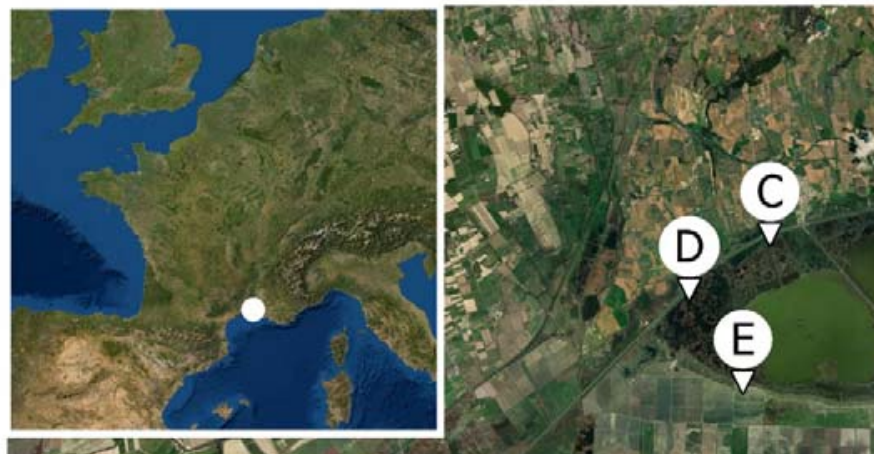

vairifin

5
5
5

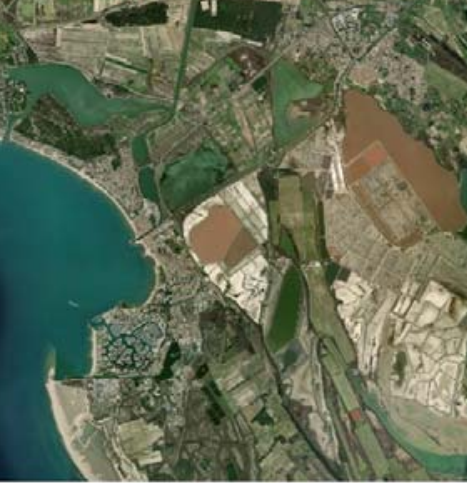

0

2.5

5
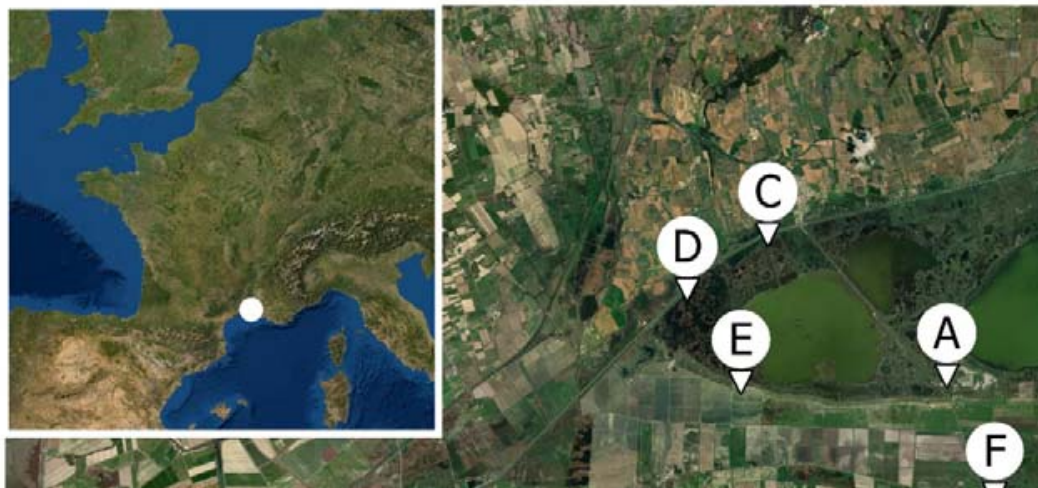

A

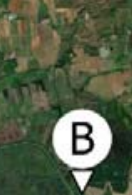

B

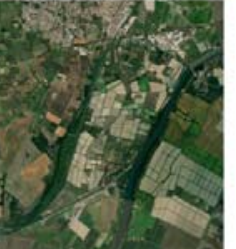

114

115 Figure 2: Study map with the geo-localization of the 8 sampling sites (A to $\mathbf{H}$ ) in the

116 Camargue wetland in South of France. The Map was created using the Free and Open 
117 Source QGIS Geographic Information System using satellite imagery from the Environmental

118 Systems Research Institute (ESRI).

119

120 In Camargue wetland, a total of 1 Culex, 17 Ochlerotatus /Aedes and 1 Anopheles

121 mosquitoes were collected in the first positive trap (F 2020/09/15) while 5 Culex, 31

122 Ochlerotatus/Aedes and 1 Anopheles mosquitoes were identified in the second trap (G

$1232020 / 09 / 22$ ). No WNV viruses were detected in these mosquitoes by RT-qPCR after an

124 individual extraction. The amount of excreta recovered on these traps was higher than it

125 would be expected by considering that they would be expelled by these mosquitoes only,

126 suggesting that a mosquito escape issue could have occurred for these traps. Culex and

127 Culiseta mosquitoes from traps tested as negative for WNV and USUV based on excreta,

128 were screened for these viruses based on an individual grinding and a pooled extraction and

129 detection procedure. These viruses were not detected in any of the 241 pools, each made of 12

130 mosquitoes.

131 We succeeded at sequencing a 341 bp WNV genomic region (GenBank accession

132 number OK489805) located on the envelope gene on one excreta sample that was tested

133 positive by RT-qPCR with the lowest Ct (sample F 2020/09/15). Phylogenetic analysis

134 revealed that the WNV identified in our study belonged to lineage 1 and was closely

135 genetically related to a WNV isolated on a symptomatic horse the $3^{\text {rd }}$ of October $2015^{13}$

136 (GenBank accession number MT863559) (Figure 3 A). Our WNV small genomic section

137 diverged from the $2015 \mathrm{WNV}$ (GenBank accession number MT863559) by 5 single

138 nucleotide polymorphism (SNPs) and from a WNV isolated in Spain on a horse in $2010^{14}$

139 (GenBank accession number JF719069) by 4 SNP (Figure 3 B). 


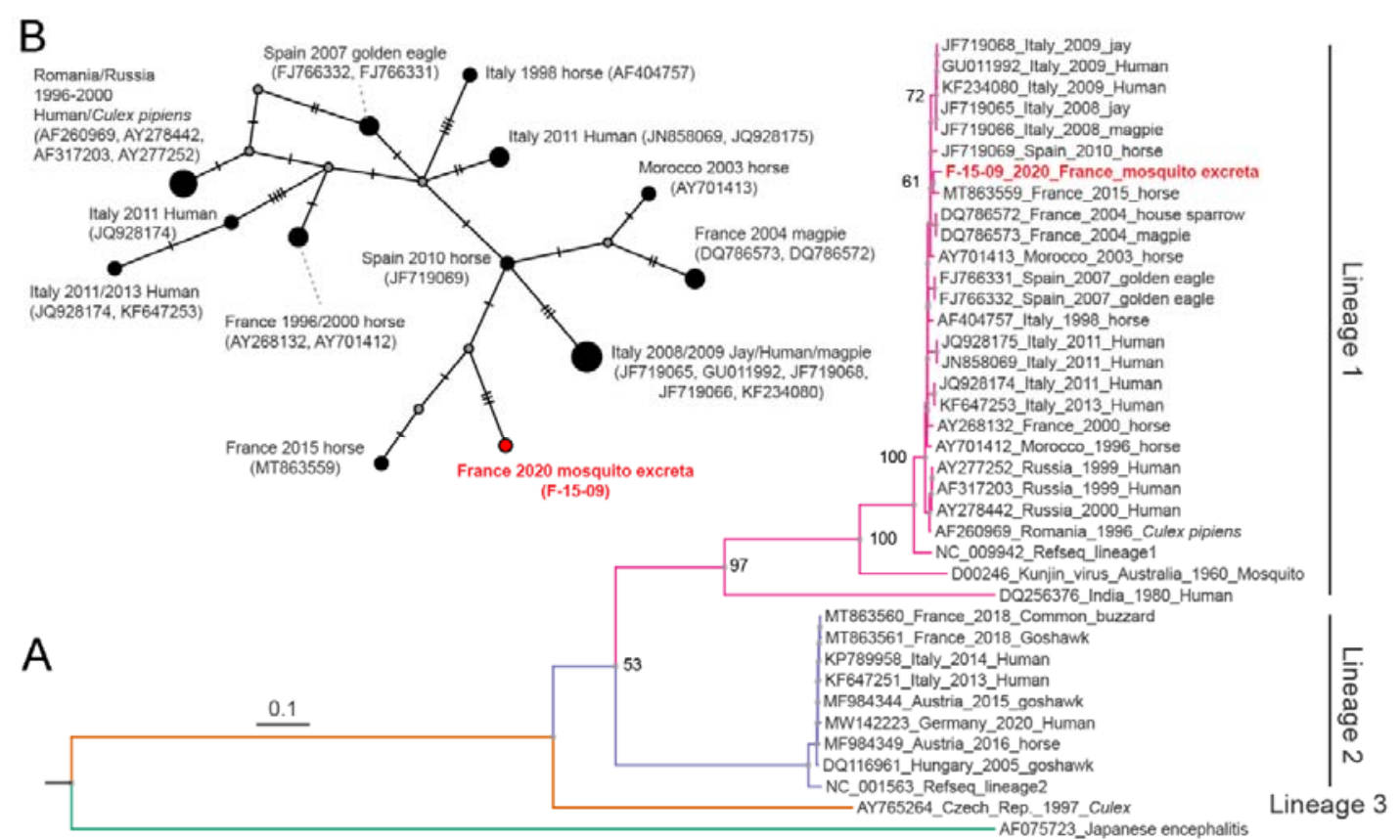

140

141 Figure 3: Molecular relationship at the intraspecific level of WNV genomes from

142 lineages 1, 2 and 3, with the virus identified in this study in mosquito excreta. (A)

143 Molecular phylogenetic tree of $38 \mathrm{WNV}$ genome, including the $341 \mathrm{bp}$ genome section

144 identified in this work. The phylogenetic analysis was performed on a curated alignment of

$14510,302 \mathrm{bp}$ by allowing gap positions within the final block. One sequence of JEV (AF075723)

146 was used as an outgroup in the phylogenetic tree. The evolutionary history was inferred using

147 a maximum likelihood method using PhyML. Bootstrap values obtained after 100 replicates

148 are shown at major nodes (percentage of replicate trees in which the associated taxa clustered

149 together). Evolutionary distances, as represented by length of branches, are expressed in

150 number of base substitutions per site. (B) Haplotype network inferred by the TCS method

151 using the same sequences as above trimmed to the length of our sequenced amplicon (341

152 bp). The size of each circle represents the frequencies of the haplotype. Mutations are shown

153 as perpendicular bars along the branches and grey small circles represent inferred unsampled

154 haplotypes. The WNV genome section identified in this study is indicated in red. 


\section{Amplicon-based metagenomic analysis on trapped mosquito excreta.}

157 The mitochondrial DNA mixture extracted from mosquito excreta samples was

158 amplified over a 460 bp mitochondrial DNA section corresponding to a sub fragment of the

159 classical Folmer cytochrome c oxidase subunit I (COI) fragment ${ }^{15}$ routinely used in

160 metabarcoding analysis. The sequencing generated a total of 3,968,028 reads across all

161 samples with a mean of $45,610\left(1^{\text {st }}\right.$ quartile: 40,$335 ; 3^{\text {rd }}$ quartile: 50,797$)$ reads per sample,

162 including the control. All paired-end reads were turned into chimera-free amplicon sequence

163 variants (ASVs) after a trimming, quality-based filtering, merging, dereplicating and

164 denoising steps ${ }^{16}$. The total number of reads was reduced to 7,752 ASVs with a median

165 abundance of 28,290 (1st quartile: 23,735 ; 3rd quartile: 33,305) per sample. Taxonomy was

166 next assigned to each ASV using blast on a database gathering Fungi, Protist, and animal COI

167 records. A total of 1,330 (17.6\%), 1,681 (22.3\%), and $53(0.7 \%)$ ASVs were assigned to the

168 Animalia, Fungi, and Protist kingdoms, respectively, and 4,486 ASVs (59.4\%) were

169 unassigned. Unassigned ASVs corresponded to off-targeted genomic amplifications or

170 assignments falling below the required $80 \%$ identity threshold. ASVs count per kingdom

171 represented $17 \%(\mathrm{~N}=406,892), 39 \%(\mathrm{~N}=924,375), 0.1 \%(\mathrm{~N}=2,292)$ and $44 \%(\mathrm{~N}=1,027,285)$

172 of the total ASVs count for the Animalia, Fungi, Protist kingdoms and unassigned ASVs,

173 respectively (Supplementary file 2). Fungi from the genera Leohumicola (class:

174 Leotiomycetes, phylum: Ascomycota), Tremella, Cryptococcus and Rhodotorula (phylum:

175 Basidiomycota) were overrepresented. The Arthropoda phylum was the most represented

176 inside the Animalia kingdom (96\% of ASVs abundance) with most ASV counts assigned to

177 the Insecta class.

178

179 Analysis of the Culicidae diversity based on trapped mosquito excreta. 
181 inside the adapter MX. The Diptera order represented $78 \%$ of ASVs abundance inside the

182 Insecta class and taxon from the Culicidae family represented $48 \%$ of ASVs counts inside the

183 Diptera order (Supplementary file 2). This was consistent with the fact that mosquitoes

184 (Culicidae) were not the only specimen recovered from the traps. One hundred and three

185 ASVs could be assigned at the species level for 14 mosquito species, i.e. Ochlerotatus

186 detritus (6 ASVs), Ochlerotatus caspius (1 ASV), Culiseta subochrea (3 ASVs), Culex

187 theileri (8 ASVs), Cx. quinquefasciatus (1 ASV), Cx. pipiens (13 ASVs), Cx. modestus (30

188 ASVs), Anopheles Hyrcanus (12 ASVs), Anopheles atroparvus (2 ASVs), An. gambiae sensu

189 lato (1 ASV), Aedes vexans (5 ASVs), Ae. albopictus (3 ASVs), and Ae. aegypti (18 ASVs).

190 Thirty-eight, 4 and 1 ASVs were assigned to the Ochlerotatus, Culex and Uranotaenia

191 genera, respectively, and 31 were assigned to the Culicidae family (Figure 4-A).

192 Shannon index was used to quantify the species diversity and richness inside each

193 sample (Alpha diversity). Alpha diversity varied from $0.66 \pm 0.75$ (mean $\pm \mathrm{SD}$, Shannon

194 index) for the Mali study site to $2.57 \pm 0.73$ for site $\mathrm{E}$ in Camargue and was not significantly

195 different across sites when considering a Holm or Bonferroni $p$-value correction (Figure 4-B).

196 The Alpha diversity in samples from Mali was significantly lower than the Alpha diversity

197 observed on all other sites in Camargue $(2 \pm 0.83$, all site from Camargue confounded, $p=$

198 0.01). Significant Alpha diversity difference was observed over time ( $p=0.03$, mixed-linear

199 regression).

200 The Culicidae compositional differences among samples (Beta diversity) were

201 significantly affected by the study area (i.e. Camargue vs Mali) with all nominal pairwise

202 comparison between Mali and Camargue sites being under the significant threshold ( $p$-value

$203<0.05$, PERMANOVA on weighted UniFrac distances), with the exception of site $\mathrm{H}(p=$

204 0.158). Beta diversity was also significatively different between site D and sites B, C, F and G 
205 in Camargue (Supplementary file 4). Culicidae species composition in site B was also

206 significantly different from site $\mathrm{A}$ and $\mathrm{E}$, and between site $\mathrm{C}$ and $\mathrm{H}$. None of these pairwise

207 comparisons were significant when considering Benjamini-Hochberg FDR correction to

208 adjust the $p$-values to account for the multiple testing. The ASV assigned to the $C x$.

209 quinquefasciatus species (feature ID: e8c12e96fda22f7f88badd812979f9cc) was

210 significatively associated to community composition differences between Camargue and

211 Mali, accounting for the differential abundance of ASVs between these study areas (Aldex2,

$212 p=0.0002$, Expected $p$ value of Welch's t test, Figure 4-C). The abundance of an ASV

213 assigned to the mosquito species $C x$. modestus (feature ID:

214 456a6b2e45a53a153c11f5a165c2c835, Supplementary file 4) was significantly over-

215 represented in sites $\mathrm{D}$ and $\mathrm{E}$ in all their pairwise comparisons described above $(p<0.05)$. At

216 the qualitative level (i.e., ASV presence/absence), ASVs assigned to An. gambiae species

217 were only detected in Mali, as expected. An. atroparvus was only detected in site $\mathrm{G}$ in

218 Camargue and Ae. vexans only in sites F and G. ASVs assigned to Ae. albopictus and Ae.

219 aegypti were detected in Camargue. These species were not identified in trapped mosquitoes

220 in this study area and are not likely to occur in Camargue, suggesting spurious ASV

221 assignments for these species.

222 Amplicon-based genomic was further used to assess the genetic diversity inside the

223 Cx. pipiens s.l. mosquito species complex. We could reveal several Culex cytochrome oxidase

224 c subunit I (COI) haplotypes circulating in Camargue (Figure 4-D). The haplotype assigned to

225 Cx. quinquefasciatus was over-represented in the Mali study site but do not branch apart on

226 the tree topology (Figure 4-D). ASVs genetic diversity was not obviously associated to $C x$.

227 pipiens pipiens and Cx. pipiens molestus cryptic forms inside the Cx. pipiens s.l. complex, as

228 revealed by the tree topology incorporating representative sequences from all forms

229 (Supplementary file 5). 
230 Mosquito species composition revealed on DNA present in mosquito excreta

231 (amplicon-based metagenomic) was compared with the species composition revealed by

232 morphological identification of trapped mosquitoes at the genus level for all sample

233 collections from Camargue. The Aedes and Ochlerotatus genera were grouped for simplicity

234 in this analysis. Mosquito species composition shared $68 \%$ similarity between both

235 identification methods when considering the symmetrical Sokal \& Michener similarity index,

236 with $111(33.6 \%)$ and $113(34.2 \%)$ matches for the presence revealed by both methods and

237 absence revealed by both methods, respectively. A total of $44(13 \%)$ and $62(18.8 \%)$

238 mismatches was revealed for the presence in metagenomic/absence in trapped mosquitoes and

239 absence in metagenomic/presence in trapped mosquitoes, respectively. The similarity dropped

240 to $51 \%$ when using the asymmetric Jaccard index that do not consider species absences in

241 both methods. Importantly, Jaccard similarity index was much lower when considering under-

242 represented mosquito species, i.e. Anopheles (Jaccard index: 20\%) or Culiseta (Jaccard index:

243 7\%) genera, as compared to the highly represented Aedes/Ochlerotatus (Jaccard index: 58\%)

244 or Culex (Jaccard index: 73\%) genera. 
A

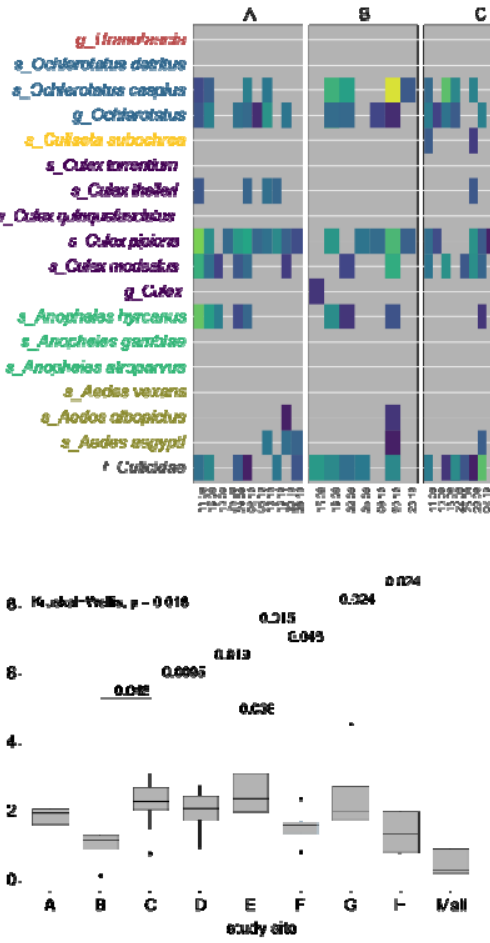

D
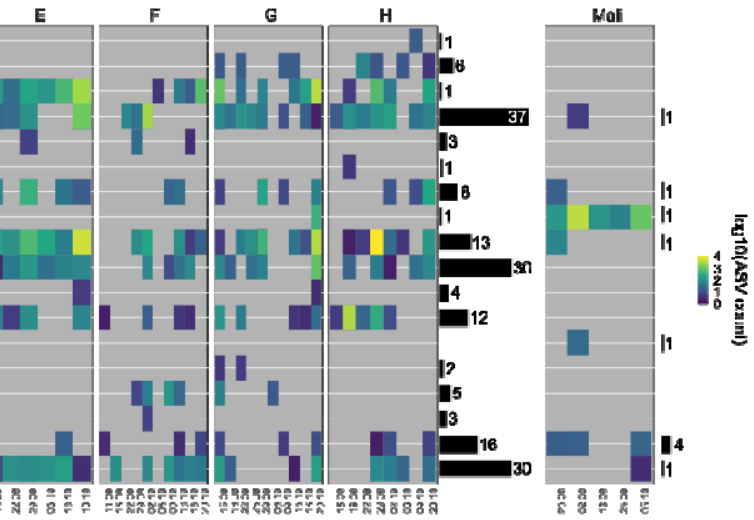

$$
\text { B }
$$
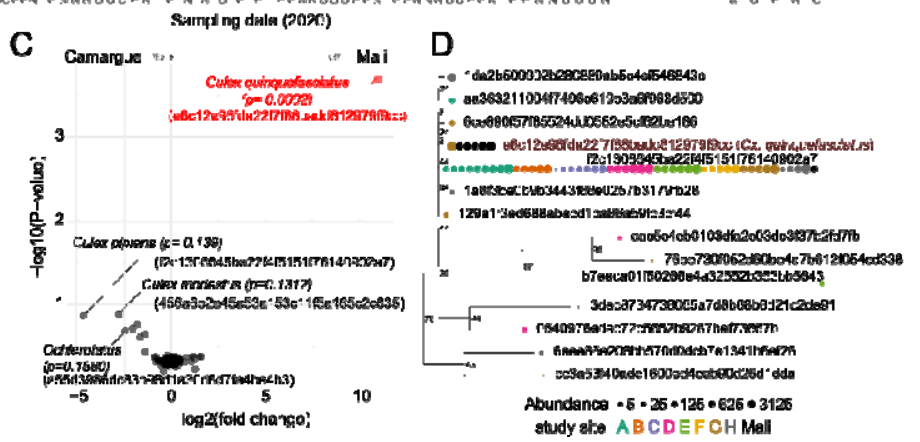

247 Figure 4: Amplicon-based metagenomic analysis of the Culicidae diversity through the

248 sequencing of trapped mosquito excreta. A- Heatmap representing the total number of ASV

249 ( $\log 10$ scale) attributed to a taxon according to sampling sites and sampling times. The

number of ASVs assigned to each taxon is represented with horizontal black bars at the right-

hand side of the graph for Camargue and Mali study sites. B-Alpha diversity (Shannon's

252

diversity index) across study sites. Kruskal-Wallis test was used to compare Shannon's

diversity across sample sites. Non-adjusted $p$ values are presented. C- Volcano plot

representing the magnitude of change (X axis) versus the $-\log 10$ expected $p$ value of Welch's

$\mathrm{t}$ test. Red represents features called as significantly differentially abundant with $p<0.05$. D-

256 Phylogenetic relationship among ASVs assigned to Culex pipiens s.l. mosquitoes. The

phylogenetic tree was derived from ultrafast bootstrap algorithm implemented in Qimme2 to 


\section{Chordata diversity revealed by amplicon-based metagenomic based on trapped}

262 mosquito excreta.

263 The Chordata phylum represented $4 \%$ of ASVs abundance inside the Animalia

264 kingdom, with most ASVs assigned to the Homo sapiens species (77\% of Chordata),

265 followed by species from the Artiodactyla order (Suidae (pigs) and Bovidae, 11\% of

266 Chordata) and birds (6\% of Chordata). At the qualitative level, Homo sapiens, Rattus

267 norvegicus, bats from the Vespertilionidae family and pigs (Sus scrofa) species were

268 identified in both Camargue and Mali study sites. ASVs assigned to a frog species

269 (Hoplobatrachus occipitalis) and the Tayassu pecari species were uniquely identified in Mali.

270 Mali is inside the frog species distribution area but the geographic distribution of pecari is in

271 South America, suggesting a spurious identification for this species. Six fish's species were

272 also detected in this study. ASVs assigned to these fish's species (class: Actinopterygii) were

273 scarce (3\% of the Chordata phylum), and Salmo salar (salmon) was the most represented and

274 present in 3 study sites in both Camargue and Mali.

275 Vertebrates typically associated to the Camargue regions were detected, such as Bos

276 taurus, Equus caballus or birds belonging to the Ardea genus (i.e. herons and egret). While

277 accounting for only $0.3 \%$ of ASVs counts inside the Animalia kingdom, ASVs assigned to the

278 Aves class were the most diverse inside the Chordata phylum with 6 birds identified as the

279 species level (i.e. Sylvia atricapilla, Motacilla flava, Hirundo rustica, Pica pica, and Corvus

280 corax from the Passeriforms order, and Gallus gallus). ASVs were also assigned to the Ardea

281 genus, Passeriformes order and Phasianidae family (Figure 5). Alpha diversity at the

282 Chordata level varied from $0.60 \pm 0.37$ (mean $\pm \mathrm{SD}$, Shannon index) for study site E to 1.28

$283 \pm 0.76$ for site B in Camargue and was not significantly different across sites but varied

284 significantly over time ( $p=0.03$, mixed-linear regression). 
286

287

288

289

290

291

292

293

294

295

296

297

298

299

300

301

302

303

304

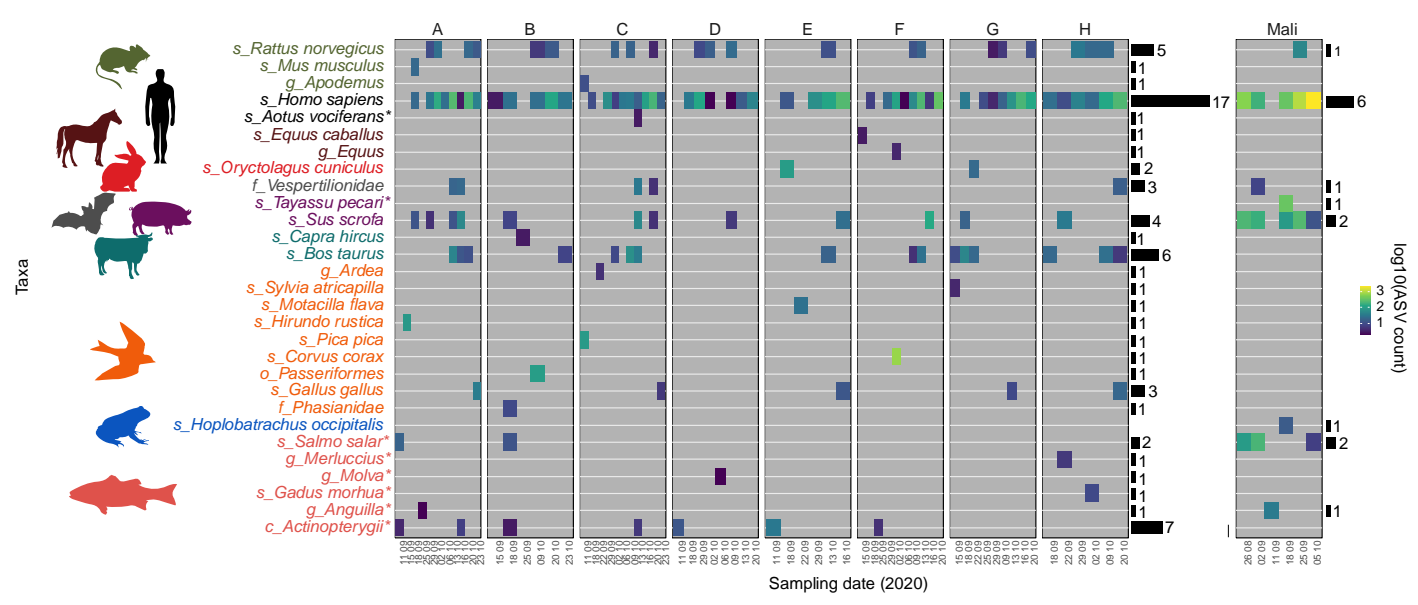

Figure 5: Vertebrate (Chordata) diversity identified based on digested blood through the

( $\log 10$ scale) attributed to a taxon according to sampling sites and sampling times. The

number of ASVs assigned to each taxon is represented with horizontal black bars at the right-

hand side of the graph for Camargue and Mali study sites.

\section{Discussion}

WNV surveillance is usually achieved through the monitoring of symptomatic cases in human and animals ${ }^{17,18}$, seroprevalence studies ${ }^{19-21}$, dead birds ${ }^{2,22}$, blood donor ${ }^{23,24}$ or virus screening in trapped mosquitoes ${ }^{25}$. High rates of asymptomatic arbovirus infections, the broad immunological cross-reactivity across flaviviruses, the difficulties to report and collect dead birds $^{26,27}$, and low arbovirus infection prevalence in mosquitoes are hindering the use of these methods to early-detect arbovirus circulation with an accurate dating of infection events. The discovery that mosquitoes with a systemic arbovirus infection can massively excrete virus $\mathrm{RNA}^{9,11}$ has open new perspectives in entomological surveillance of viruses ${ }^{10,28,29}$. Indeed, virus identification in trapped mosquito excreta considerably alleviates the processing charge and costs associated with entomological surveillance by extending the period between mosquito trap collections and making optional the steps of mosquito species identification, 
305 pooling, and testing. This non-invasive method that does not require strong entomological

306 knowledge can also be extended to monitor mosquito-borne parasites ${ }^{30,31}$.

307 Here, we succeeded at identifying WNV circulation in Camargue over a short 6-week

308 period without the need to process any mosquito. This is only the third time that WNV have

309 been detected in mosquitoes in this region since $196^{13,32,33}$ despite important efforts to search

310 for infected vectors, including after epidemics. The sequencing of a short genomic region on

311 the envelope gene indicated its affiliation to WNVs from lineage 1 (Western Mediterranean

312 clade) that circulated in Southern Europe in the last 20 years ${ }^{13}$. These viruses can be

313 maintained locally from one year to another in enzootic cycles that involve resident bird

314 species and ornithophilic mosquitoes by overwintering in hibernating infected adult

315 mosquitoes $^{34,35}$ or in bird species with chronic infection ${ }^{36,37}$. Another hypothesis would be

316 recurrent introductions through migratory birds that would carry the viruses northward during

317 spring migration from African wintering places where these viruses are endemic, or from

318 West Europe in late summer when birds return to Africa. Implementing active WNV

319 surveillance in paths and resting places of migratory birds in Europe and Africa would help to

320 resolve the mechanisms of WNV maintenance or resurgence in Europe. Unfortunately, no

321 infected mosquitoes were recovered from the traps positive for WNV based on detection on

322 mosquito excreta due to mosquito escape issues that occurred at the beginning of this study.

323 Complete genome virus sequencing and WNV infection prevalence would have been easier to

324 achieve by processing trapped mosquitoes as a second intention. In case of a mosquito escape

325 due to a trap failure or a faulty handling, arbovirus RNA and trapped mosquito DNA can still

326 be recovered in the excreta with our system. For instance, we could assess the mosquito

327 species diversity and composition in Mali without an access to the trapped mosquitoes.

328 Mosquito excreta collected on filter papers are also easy to transport and store from a remoted

329 field site to the laboratory even in an absence of cold storage. 
We succeeded to have a global view of the mosquito species composition and

331 abundance in different sampling sites. Mosquito species emblematic to Camargue such as

332 Ochlerotatus caspius and Culex pipiens were identified via their DNA contained in their

333 excreta in Camargue but not in Mali. Taxonomic identification is limited with our method to

334 the genetic diversity that can be captured and amplify over a short mitochondrial sequence.

335 Here, taxonomic identifications are biased toward arthropods because we used a degenerated

336 primer set specifically designed to amplify arthropods ${ }^{38,39}$. The use of primers targeting

337 vertebrates' mitochondrial DNA on the same target might further increase our ability to

338 identify the diversity of vertebrate species on which mosquitoes fed prior to be trapped ${ }^{40}$.

339 These limitations might have explained the spurious or unexpected species identifications in

340 this study. Aedes albopictus and Ae. aegypti COI sequences might share a strong homology

341 on our target region with other Aedes species in Camargue that are not present in our

342 database. The identification of fish species in this study might have resulted from any DNA

343 contamination from the environment (e.g. food contamination), laboratory reagents or kits ${ }^{41}$.

344 ASVs assigned to these fish species were scarce and filtering out low-abundant and/or low-

345 prevalent features might improve false-identifications, with the trade-off of losing species

346 identification. The Cx. pipiens s.l. complex has two recognized forms - pipiens and molestus -

347 that exhibit behavioral and physiological differences and can form hybrids. Culex pipiens

348 pipiens feed preferentially on birds, and Cx. pipiens molestus on humans but both can

349 transmit $\mathrm{WNV}^{42,43}$. Our method could reveal genetic diversity inside the species level but

350 could not associate $C x$. pipiens s.l. COI haplotypes to one of these biotypes. Failure to

351 discriminate these forms inside the complex using mitochondrial DNA has been previously

352 described $^{44,45}$. Additional nuclear genomic regions could be targeted in the metagenomic

353 scheme to further discriminate these species inside the complex ${ }^{43}$ or to non-invasively screen

354 for known molecular signatures (e.g. insecticide resistance). 

mosquito excreta. Determining the reservoir role of local bird species for WNV is not straightforward due to the challenging task of obtaining blood samples from animal for diagnoses purposes. It usually involves wild bird captures that are difficult to operationalize or blood-meal analysis of trapped mosquitoes prior to implement experimental infection studies $^{46,47}$. Blood-fed mosquitoes can be accidentally captured in $\mathrm{CO} 2$ baited traps or be

361 trapped during their quest to complete their blood-meals. It is also possible to specifically

362 target blood-engorged mosquitoes in their resting sites, but this method implies an active

363 prospection and is difficult to implement for exophilic species. Our method captures

364 vertebrate blood as it is incrementally digested by trapped mosquitoes without the need to

365 process mosquitoes during their digestion stage. Blackcaps (Sylvia atricapilla), Barn

366 swallows (Hirundo rustica), Yellow wagtails (Motacilla flava) and herons or egrets identified

367 in this study were previously listed as bird species potentially involved in the introduction,

368 amplification and spread of WNV in Camargue ${ }^{48}$. They are all bird species present in

369 Camargue during the mosquito season that migrate in West Africa during winter. WNV

370 antibodies have been detected in most of these species ${ }^{49}$. WNV has also been isolated from

371 common magpies (Pica pica) $)^{50}$ in 2004 in Camargue. Corvidae are sedentary birds that are

372 potential hosts to amplify the virus and to bring it closer to the urban areas. Our method

373 cannot directly link a virus collected in trapped mosquitoes to a vertebrate host. However, it

374 can reveal trophic preferences and the range of potential hosts involved in WNV introduction

375 and amplification in different areas. Ecological factors associated with WNV outbreaks are

376 not yet fully understood. Increased host diversity can either reduce risk of virus emergence by

377 diluting the abundance of a vector or a reservoir host inside a community ${ }^{51}$ or increase the risk

378 if the abundance of a vector is a function of vertebrate host diversity ${ }^{52}$. Extending the 
entomological survey over several diverse locations and seasons might help to assess if bird or mosquito species diversity and composition can influence the risk of WNV emergence. Costs and logistic constraints are impeding the implementation of nation-wide entomological surveillance programs worldwide. Here, we used both the RNA and DNA genomic materials contained into excreta from trapped mosquitoes to (i) non-invasively survey the emergence and spread of WNV, (ii) monitor mosquito species diversity without the processing of mosquitoes and (iii) to invade ecological network underlying WNV emergence by identifying vertebrate animals on which trapped mosquitoes fed before to be captured, using a single and easy to implement amplicon-based metagenomic procedure. Our costeffective strategy can be considered as a complementary early-warning tool to detect the circulation of mosquito-borne pathogens affecting human health or to non-invasively survey the presence of these pathogens in remote areas without health-care system.

\section{Methods}

\section{D-printed BG sentinel mosquito trap modification}

We created a 3D-printed adapter (MX adapter) to increase mosquito longevity in BG Sentinel (BGS, Biogents AG, Regensburg, Germany) traps, inspired from the device designed by Timmins D.R. and colleagues ${ }^{53}$. Briefly, our system is composed of a 120-mm high and 87/90 $\mathrm{mm}$ (inside/outside) diameter cylinder with a removable cap that is inserted into the depressurized BGS catch pipe. The MX adapter is attached beneath the intake funnel and is replacing the original catch bag. The original catch bag was cut at the bottom to house the MX adapter (Figure 1A). The MX adapter is traversed longitudinally by an airflow piping to allow air depressurization inside the catch pipe, and thereby allowing aspiration at the top of the BGS intake funnel via the fan located underneath the system. The airflow piping is cut with its upward pointing section sealed with mesh fabric to prevent mosquito escape from the 
404 device while letting the air flows through. A mosquito feeder, filled with a cotton ball soaked

405 in $10 \%$ sugar water, is attached to the inner side of the cylinder. The MX adapter has a

406 removal bottom on which a filter paper (Whatman, grade 3, ref. 1003-917) can be placed

407 when the system is set in mosquito catching mode. A vertical sliding gate can be used to seal

408 the system during transport when the bottom has been removed (optional) (Figure 1B). The

409 MX adapter provide a safe and moisturized enclosure for trapped mosquito with an easy

410 access to sugar. Mosquito excreta can be easily collected on the filter paper at the bottom of

411 the adapter. First version of BGS traps were used in this study but our device can be adapted

412 to BG-Sentinel 2 and CDC light traps (Supplementary figure 1). The MX adapter was created

413 on Fusion 360 (AutoDesk) and 3D printed in either PLA or PETG on a Sigmax R19 (BCN

414 3D). MX adapter 3D files (.stl format) are provided in supplementary file 3 under the Creative

415 Commons (CC) license BY-NC-SA.

417 Study area and samples collection

418 The study was carried out in a $\sim 130 \mathrm{~km}^{2}$ area in Camargue (Figure 2), a large wetland in the

419 Southeast of France with a temperate Mediterranean climate located inside the Rhone river

420 delta. The Camargue is a nature reserve that hosts large populations of resident and migratory

421 birds and is famous for its white horses and black bulls. West Nile virus transmission has

422 been repeatedly reported in Camargue for several years ${ }^{50,54,55}$. BGS traps were placed at the

423 ground level at 8 sites nearby the "Marais du Charnier", each separated by few kilometers.

424 Adult mosquitoes were captured over 6 weeks from the $11^{\text {th }}$ of September 2020 to the $23^{\text {rd }}$ of

425 October 2020, at the end of the mosquito season, using BGS modified with the MX adapter,

426 as described previously. Mosquito captures were conducted over 3 to 4 consecutive days, with

427 traps collection and reconditioning for a new mosquito trapping session occurring twice a

428 week. Carbon dioxide was provided as a mosquito attractant for every BGS traps. Pressurized 
carbon dioxide bottles and $12 \mathrm{~V}$ power packs were used to operate over time. During trap

430 collection, the MX adapter was removed from the BGS and maintained 5 minutes on a sealed

431 plastic box filled with dry ice to knock down mosquitoes. Knock down mosquitoes were then

432 transferred to $50 \mathrm{~mL}$ tube and transported alive to the laboratory. This mosquito collection

433 procedure avoids mosquitoes to escape from the device and was implemented late in our

434 mosquito collection session (after the end of September) in response to mosquito escape

435 issues coming from the fields. Filter paper covered with mosquito excreta were placed into

436 annotated sealed plastic bags and transported at ambient temperature to the laboratory. Once

437 in the laboratory, mosquitoes and filter papers were stored at $-20^{\circ} \mathrm{C}$ until the extraction

438 procedure. Adult mosquitoes were identified morphologically in the laboratory at the genus

439 level and sorted by genus, trap, and collection date. One trap was implemented in Gao, Mali,

440 from the 26th of August 2020 to the 5th of October 2020 in continuous operation with 6

441 sampling over this period. No mosquito was brought back to the lab and only mosquito

442 excreta were analyzed for these samples.

443

444 RNA/DNA extraction

445 RNA/DNA extraction from filter papers with mosquito excreta

446 Virus detection was first conducted on mosquito excreta collected on filter papers. Briefly,

447 filter papers were coiled and placed individually at the bottom of $14 \mathrm{~mL}$ plastic tubes (Falcon, 448 ref: 352059 ) before to be soaked in $3 \mathrm{~mL}$ of Lysis buffer RAV1 (NucleoSpin 96 virus core

449 kit, Macherey $\square$ Nagel, Düren, Germany) for 5 minutes. Ten microliters of phage MS2 (RNA)

450 were added to each tube as an internal extraction control. Filter papers were then manually

451 grinded with a $5 \mathrm{~mL}$ pipette. Several $1 \mathrm{~mm}$ diameter holes were drilled in the transparent caps

452 of the $14 \mathrm{ml}$ tubes to create a colander, and then clipped on each tube. Closed tubes were

453 placed upside-down on a larger $50 \mathrm{~mL}$ tube (with the colander cap placed downward, at the 
454 bottom of the $50 \mathrm{~mL}$ tube) and centrifuged $5 \mathrm{~min}$ at 3,000 rpm. Flowthroughs were collected

455 and mixed with $3 \mathrm{~mL} 96-100 \%$ ethanol before to be loaded on NucleoSpin Virus Columns in

456 several steps. RNA/DNA extraction was performed as indicated by the manufacturer

457 indications. Eluates were kept at $-20^{\circ} \mathrm{C}$ until use.

458

RNA/DNA extraction from captured mosquitoes

460 Virus detection in large number of samples is time consuming and cost prohibitive, especially

461 when low infection rates are expected in mosquito populations. Here, we implemented a

462 strategy based on the simultaneous grinding of 96 individual mosquitoes in a 96-well plate

463 inspired from Holleley C. and Sutcliffe A. MR4 protocol $^{56}$, followed by an extraction and

464 detection in pool of 12 mosquitoes (one lane of a plate). This strategy drastically reduces

465 labor and costs associated to virus screening, as compared to virus detection in individual

466 mosquitoes, while still providing the opportunity to sequence or isolate virus in single

467 mosquito in a second intention, and to obtain accurate prevalence estimates. Briefly, Culex

468 mosquitoes were placed individually in each well of a shallow conical bottom 96-well plate

469 with $80 \mu \mathrm{L}$ of buffer composed of $1.3 \mathrm{~mL}$ of Proteinase $\mathrm{K}$ resuspended in proteinase buffer

470 according to the manufacturer instructions (NucleoSpin 96 virus core kit, Macherey-Nagel) in

$4719 \mathrm{~mL}$ PBS. A male Ae. albopictus infected with the insect specific Aedes flavivirus (AEFV)

472 was add on well position H12 of each plate as an internal extraction control. All mosquitoes

473 from a plate were simultaneously grinded for 5 minutes by moving up and down and rocking

474 vigorously a disposable bacterial colony replicator tool. Plates were then incubated at $70^{\circ} \mathrm{C}$

475 for 1 hour with the bacterial colony replicator tool left on the top of the plate. After a short

476 grinding step post-proteinase $\mathrm{K}$ digestion, the replicator tool was discarded and $15 \mu \mathrm{L}$ of each

477 well from a same plate line were pooled. Plates with individually grinded mosquitoes were

478 stored at $-20^{\circ} \mathrm{C} .500 \mu \mathrm{L}$ of AVL lysis buffer (QIAamp Viral RNA kit, Qiagen, Hilden, 
479 Germany) was added to each pool with $5 \mu \mathrm{L}$ of phage MS2 as an additional internal

480 extraction control. After 10 minutes of incubation at room temperature, $500 \mu \mathrm{L}$ of $100 \%$

481 ethanol was added to each pool and RNA was extracted according to the manufacturer

482 protocol (QIAamp Viral RNA kit, Qiagen).

483

484 Viruses and Plasmodium spp. detection

485 Detection of WNV and USUV genomic RNA was performed with a one-step reverse

486 transcription quantitative polymerase chain reaction (RT-qPCR) assay. The multiplexed RT-

487 qPCR was performed using the SuperScript one-step RT-PCR Platinum® Taq Mastermix

488 (Invitrogen, Cergy Pontoise, France), MS2 primers and probe (21347398), or either WNV or

489 USUV specific primers and probe (Supplementary table 1) using $10 \mu \mathrm{L}$ of buffer $2 \mathrm{x}, 0.8 \mu \mathrm{L}$

490 of forward and reverse primers, $0.3 \mu \mathrm{L}$ of probe, $0.8 \mu \mathrm{L}$ of enzyme and $2.3 \mu \mathrm{L}$ of RNase-free

491 water for one reaction, with the following cycling protocol : $48^{\circ} \mathrm{C}$ for $30 \mathrm{~min}, 95^{\circ} \mathrm{C}$ for 10

$492 \mathrm{~min}, 50$ cycles at $95^{\circ} \mathrm{C}$ for $15 \mathrm{sec}, 60^{\circ} \mathrm{C}$ for $1 \mathrm{~min}$ ). Amplification was performed on a CFX

493 real-time thermocycler (Bio-Rad, Hercules, CA, USA). Concerning the detection of AEFV in

494 infected male Ae. albopictus internal controls (extraction procedure for trapped mosquitoes in

495 pools), virus genomic RNA was first reverse transcribed to complementary DNA (cDNA)

496 with random hexamers using M-MLV Reverse Transcriptase (Life Technologies, Inc.,

497 Gaithersburg, Maryland, USA) according to the manufacturer's instructions. cDNA was

498 amplified by 35 cycles of PCR using the corresponding set of primers described in

499 Supplementary table 1 and amplicons were visualized by electrophoresis on a 1\% agarose gel.

500 Plasmodium spp. detection was performed using $10 \mu \mathrm{L}$ of $2 \mathrm{x}$ SYBR Green PCR Master Mix

501 (Thermofisher scientific, Waltham, USA), $0.5 \mu \mathrm{L}$ of forward and reverse $16 \mu \mathrm{M}$ primers

502 (Supplementary table 1), $4 \mu \mathrm{L}$ of RNase-free water and $5 \mu \mathrm{L}$ of DNA extract for one reaction

503 using the following cycling protocol: $95^{\circ} \mathrm{C}$ for $5 \mathrm{~min}, 45$ cycles at $95^{\circ} \mathrm{C}$ for $15 \mathrm{sec}, 60^{\circ} \mathrm{C}$ for 1 
504 min. Amplification was performed on a LightCycler (Roche Diagnostics, Mannheim,

505 Germany).

506

\section{$507 \quad$ Virus sequencing}

508 We sequenced WNV genome based on the tiling amplicon-based sequencing (PrimalSeq)

509 method developed by Quick J. ${ }^{57}$, and Grubaugh N.D. ${ }^{58}$ and colleagues. Briefly, PrimalSeq

510 protocol generates overlapping amplicons of $\sim 400$ base pairs from 2 multiplexed PCR

511 reactions to generate sufficient templates for subsequent high-throughput sequencing. We

512 used the multiplex primer scheme developed for WNV lineage 1 that have been validated by

513 Grubaugh N.D. et.al. ${ }^{58}$. To identify the WNV lineage before whole genome sequencing, we

514 sequenced in first intention 5 overlapping genomic sections covering the envelop gene with a

515 set of primers degenerated to amplify WNV lineages 1 and 2. Illumina Nextera ${ }^{\circledR}$ universal

516 tails sequences were added to the 5' end of each of these primers to facilitate the library

517 preparation by a two-step PCR approach (WNV lin.1 \& 2, Supplementary table 1). Purified

518 RNAs from excreta samples positive for WNV in Q-RT-PCR were first reverse transcribed to

519 complementary DNAs (cDNAs) with random hexamers using M-MLV Reverse Transcriptase

520 (Life Technologies) according to the manufacturer's instructions. For the multiplex PCR

521 reactions, $5 \mu \mathrm{l}$ of cDNA was used in a $20 \mu \mathrm{l}$ reaction mixture made of $5 \mu \mathrm{l}$ of Hot START $5 \mathrm{X}$

522 BIOamp DNA Polymerase mix (Biofidal, Lyon, France), $4 \mu \mathrm{l}$ of forward and reverse primer

$523 \operatorname{mix}$ at $10 \mu \mathrm{M}$, and $11 \mu \mathrm{l}$ of water. The thermal program was: $10 \mathrm{~min}$ of polymerase activation

524 at $96^{\circ} \mathrm{C}$ followed by 35 cycles of (i) $30 \mathrm{sec}$ denaturing at $96^{\circ} \mathrm{C}$, (ii) $30 \mathrm{sec}$ annealing at $62^{\circ} \mathrm{C}$

525 and (iii) 1 min extension at $72^{\circ} \mathrm{C}$, followed by a final incubation step at $72^{\circ} \mathrm{C}$ for 7 min to

526 complete synthesis of all PCR products. A 15 cycles PCR was then performed using

527 Nextera ${ }^{\circledR}$ Index Kit - PCR primers, that adds the P5 and P7 termini that bind to the flow cell

528 and the dual 8 bp index tags. Resulting amplicons were purified with magnetic beads 
529 (SPRIselect, Beckman Coulter), quantified by fluorometric quantification (QuantiFluor®

530 dsDNA System, Promega) and visualized on QIAxcel Capillary Electrophoresis System

531 (Qiagen). Libraries were sequenced on a MiSeq run (Illumina) using MiSeq v3 chemistry

532 with $300 \mathrm{bp}$ paired-end sequencing.

533 After demultiplexing, trimmomatic v0.33 ${ }^{59}$ was used to discard reads shorter than 32

534 nucleotides, filter out Illumina adaptor sequences, remove leading and trailing low-quality

535 bases and trim reads when the average quality per base dropped below 15 on a 4-base-wide

536 sliding window. Reads were aligned to the WNV lineage 1 reference genome (RefSeq entry:

537 NC_009942) with bowtie2 v.2.1.0 $0^{60}$. The alignment file was converted, sorted and indexed

538 using Samtools v0.1.19. Coverage and sequencing depth were assessed using bedtools v2.17. $0^{62}$. The consensus sequence was generated with Ivar $^{58}$ with default options.

\section{Phylogenetic analyses}

542 A background set of 36 full-length WNV genome sequences across different lineages was

543 obtained from GenBank. One full-length genome sequence of Japanese Encephalitis Virus

544 was also downloaded to be used as an outgroup in the phylogenetic tree. Genome sequences

545 were aligned using Clustal Omega and curated by Gblocks software implemented in the

546 seaview v.5.0.4 interface ${ }^{63}$ by allowing gap positions within the final block and less strict

547 flanking positions before to generate the best-scoring maximum-likelihood (ML) tree with

548100 bootstrap replicates with PhyML (15980534). The GTR + I + G nucleotide substitution

549 model was chosen based on the lowest Akaike Information Criterion (AIC) value using the

550 Smart Model Selection in PhyML (SMS) software ${ }^{64}$. Phylogenetic trees were visualized using

551 the ggtree R package ${ }^{65}$. All sequences were trimmed to the length of our sequenced amplicon

552 (341 bp) before to be imported into the PopArt program ${ }^{66}$ to create a TCS haplotype

553 network $^{67}$. 


\section{Amplicon-based metagenomic}

556 The amplicon-based metagenomic method implemented here was performed on mosquito 557 excreta eluates post NucleoSpin Virus RNA/DNA extraction. Even though the NucleoSpin

558 Virus Columns kit is optimized to extract virus RNA/DNA, the eluate also contained non-

559 viral RNA/DNA. Virus detection, sequencing and metagenomic approaches were performed

560 on the same eluates to facilitate the procedure. A $460 \mathrm{bp}$ mitochondrial DNA section

561 corresponding to a sub fragment of the classical Folmer cytochrome c oxidase subunit I (COI)

562 fragments ${ }^{15}$, was amplified with universal primers BF2/BR2 ${ }^{68}$ (Supplementary table 1) that

563 are routinely used for macroinvertebrate monitoring ${ }^{38,39}$. Illumina Nextera ${ }^{\circledR}$ universal tails

564 sequences were added to the 5' end of each of these primers to facilitate library preparation,

565 as described above for the WNV tiling amplicon-based sequencing procedure. PCR

566 amplifications, library preparation and sequencing were made according to the same protocol

567 described above for WNV sequencing excepting for the annealing temperature that was set to

$56850^{\circ} \mathrm{C}$ for $30 \mathrm{sec}$. All raw sequences have been deposited in the NCBI database under the

569 NCBI BioProject number PRJNA768434.

570

571 Taxonomic identification and diversity indices

572 Demultiplexed fastq sequences were imported to QIIME2 version 2021.2 for

573 bioinformatics analyses. The qiime2-dada2 pipeline ${ }^{16}$ was used for turning paired-end fastq

574 files into merged reads, filtering out Illumina adapters, denoising and removal of chimeras

575 and filtering out replicates. Taxonomic assignment was carried out for the amplicon sequence

576 variants (ASVs) using the qiime2-feature-classifier classify-consensus-blast plugin using a

577 database of 1176764 sequences gathering Fungi, Protist, and Animal COI records, recovered

578 from the Barcode of Life Database Systems (BOLD) the $7^{\text {th }}$ March 2021. The database was 
579 built according to the step-by-step tutorial provided by Devon O'Rourke ${ }^{69}$. Only sequences

580 within our primer coordinates were retained in the final database. A percentage identity

581 threshold of $90 \%$ was used to assign a taxonomy to an ASV.

582 Shannon's diversity index was used to assess Culicidae and Chordata species richness

583 and evenness (relative abundance of species inside a sample), i.e. Alpha diversity, across time

584 and sampling sites. Kruskal-Wallis test was used to compare Shannon's diversity across

585 sample sites using the compare_means function implemented in the ggpubr R package ${ }^{70}$.

586 Bonferroni $p$-value correction was used to account for the multiplicity of tests. A linear

587 mixed-effects model implemented with the lme4 R package ${ }^{71}$ was used to assess differences

588 in Alpha diversity across time. A random effect was implemented on the study site. Data were

589 rarified to 150 (Culicidae analysis) and 50 (Chordata analysis) reads per sample, before to

590 assess the compositional differences among samples (Beta diversity) using a permutational

591 analysis of variance (PERMANOVA) on weighted UniFrac distances that compare species

592 compositions considering both the relative abundance and phylogenetic distances among

593 species. Benjamini-Hochberg FDR correction was used to adjust the $p$-values to account for

594 the multiple testing. Phylogenetic distances between species from the Culicidae family and

595 Chordata phylum were calculated with IQ-TREE ${ }^{72}$ with the ultrafast bootstrap algorithm and

596 automatic model selection, as implemented in the Qiime2 phylogeny iqtree-ultrafast-bootstrap

597 plugin. Differential ASVs abundances between sampling sites were compared on a non-

598 rarefied dataset using the q2-aldex 2 differential abundance package, by modelling the data as

599 a log-ratio transformed probability distribution rather than as counts ${ }^{73}$. The R package

600 phyloseq $^{74}$ was used to represent phylogenetic relationship among ASVs assigned to Culex

601 pipiens mosquitoes. All further diversity metrics implemented in Qiime 2 can be accessed by

602 running Qiime2 diversity commands on data provided on Supplementary files 4. 
604 (asymmetrical) were used to compare the Culicidae fauna composition revealed based on 605 trapped mosquitoes identified morphologically and amplicon-based metagenomic on the 606 corresponding mosquito excreta for all sample collections from Camargue at the genus level.

607 Considering a contingency table of binary data such as $\mathrm{n} 11=\mathrm{a}, \mathrm{n} 10=\mathrm{b}, \mathrm{n} 01=\mathrm{c}$ and $\mathrm{n} 00=\mathrm{d}$,

608 the Sokal \& Michener index was computed as follow: $\mathrm{s}=(\mathrm{a}+\mathrm{d}) /(\mathrm{a}+\mathrm{b}+\mathrm{c}+\mathrm{d})$, and Jaccard

609 index as follow: $\mathrm{s}=\mathrm{a} /(\mathrm{a}+\mathrm{b}+\mathrm{c})$. The latter is an asymmetrical index that does not treat double

610 zero (d) in the same way as double presences (a) as a reason to consider samples similar.

611 All statistical analyses were performed in the statistical environment R. Figures were

612 made using the package ggplot $2^{75}$ and the Tidyverse ${ }^{76}$ environment. The Map was created

613 using the Free and Open Source QGIS Geographic Information System using satellite

614 imagery from the Environmental Systems Research Institute (ESRI).

615

\section{Funding statement}

617 This study received funding from the Direction Générale de l'Armement (grant no PDH-2-

618 NRBC-2-B-2113) and from the Direction de la Formation de la Recherche et de l'Innovation

619 (Grant MX, DFRI). The contents of this publication are the sole responsibility of the authors.

620 The funders had no role in study design, data collection, and interpretation, or the decision to

621 submit the work for publication.

622

\section{Acknowledgments}

624 We are grateful to Laurie-Lou Weghel for her interest and support throughout this project. We 625 also thank Frédéric Jean (EID Méditerranée) for his thorough involvement on the field work 626 and Nicolas Benoit for his help regarding the molecular detection of Plasmodium spp. We 627 also thank Clément Gendrot and Sylvain Buffet for their help at designing and printing the 
628 first version of the adapter MX, it all started from there. We are also thankful to the Qiime2

629 contributors and community for providing such an easy, enjoyable, and pre-built access to

630 metagenomic analyses.

631

\section{Conflict of interests}

633 The authors declare that there is no conflict of interest regarding the publication of this article.

634

\section{Data availability}

636 Amplicon-based metagenomic raw sequencing data are accessible under the NCBI BioProject

637 number PRJNA768434. The WNV genomic section sequenced in this project is accessible

638 under the GenBank accession number OK489805. Qiime2 artifacts with all amplicon-based

639 data are available in Supplementary file 4.

640

641 Supplementary information

642 Supplementary table 1: Molecular amplification systems used in this study.

643 Oligonucleotides sequences (primers and probes) are presented with their corresponding

644 species, gene targets and amplicon sizes. Illumina Nextera ${ }^{\circledR}$ transposase sequences that have

645 been added to primers during the PCR amplification step of the metagenomic method are

646 represented in green.

647

648 Supplementary file 1: Mosquito species inventory. Number of mosquitoes identified

649 morphologically at the genus level in each trap. Anopheles mosquitoes were further identified

650 at the species or species complex level. 


\section{Supplementary file 2: Interactive representation of the metagenome of trapped}

653 mosquito excreta displayed with a Krona chart. The number 2,360,844 represents the total

654 number of ASV counts across all samples. Taxonomy nodes are shown as nested sectors

655 arranged from the top level of the hierarchy at the center and progressing outward.

656 Navigational controls are at the top left, and details of the selected node are at the top right.

658 Supplementary file 3: MX adapter 3D files in .stl format. MX adapter is under the Creative

659 Commons (CC) license BY-NC-SA (Licensees may copy, distribute, display, and make

660 derivatives only for non-commercial purposes and by giving credits to the authors).

661

662 Supplementary file 4: Archive comprising (i) Qiime2 code that was used in the amplicon-

663 based metagenomic analysis pipeline, (ii) the data base with sequences recovered from public

664 repositories (bold_COI_seqs_ref_db.qza and bold_COI_taxa_ref_db.qza), (iii) alpha-

665 rarefaction analysis (alpha_rarefaction_curves.qzv), (iv) metadata file associated to the data

666 (COI-metadata.txt), (v) denoised features (rep-seqs-trim.qza/.qsv) and their associated

667 taxonomy (taxonomy_blast-trim_80identity.qza/.qsv). PERMANOVA results for Culicidae

668 (weighted-unifrac-study_site_significance_Culicidae.qzv) and Chordata (weighted-unifrac-

669 study_site_significance_Chordata.qzv) are provided as well as Aldex2 differential abundance

670 analysis between sites from Camargue (Aldex_figures.html). All further diversity metrics

671 implemented in Qiime2 can be accessed by running Qiime2 diversity commands on data

672 provided. All .qsv files can be easily loaded on the Qiime2 visualizer at

673 https://view.qiime2.org.

674

675 Supplementary file 5: A phylogenetic tree displaying the intra Cx. pipiens s.l. complex

676 genetic diversity revealed by our method relative to the $C x$. pipiens and Cx. molestus forms. 
677 Haplotypes revealed in our study are represented with their feature ID in dark red. GenBank

678 accession numbers of sequences representative to $C x$. quinquefasciatus, Cx. pipiens form

679 molestus and Cx. pipiens form pipiens are represented in green, blue, and black, respectively.

680 The evolutionary history was inferred using a maximum likelihood method using PhyML.

681 The GTR + I nucleotide substitution model was chosen based on the lowest Akaike

682 Information Criterion (AIC) value using the Smart Model Selection in PhyML (SMS)

683 software. Evolutionary distances, as represented by length of branches, are expressed in

684 number of base substitutions per site.

685

Supplementary figure 1: Picture of the MX adapter fit to a CDC light trap.

\section{References}

689 1. Gould, E. A. Evolution of the Japanese encephalitis serocomplex viruses. Curr Top

690 Microbiol Immunol 267, 391-404 (2002).

691 2. Weissenböck, H., Hubálek, Z., Bakonyi, T. \& Nowotny, N. Zoonotic mosquito-borne

692 flaviviruses: worldwide presence of agents with proven pathogenicity and potential candidates

693 of future emerging diseases. Vet Microbiol 140, 271-280 (2010).

694 3. Campbell, G. L., Marfin, A. A., Lanciotti, R. S. \& Gubler, D. J. West Nile virus.

695 Lancet Infect Dis 2, 519-529 (2002).

696 4. Hubálek, Z. \& Halouzka, J. West Nile fever--a reemerging mosquito-borne viral

697 disease in Europe. Emerg Infect Dis 5, 643-650 (1999).

698 5. Calistri, P. et al. Epidemiology of west nile in europe and in the mediterranean basin.

699 Open Virol J 4, 29-37 (2010).

700 6. Joubert, L. et al. [Epidemiology of the West Nile virus: study of a focus in Camargue.

701 IV. Meningo-encephalomyelitis of the horse]. Ann Inst Pasteur (Paris) 118, 239-247 (1970).

702 7. Tsai, T. F., Popovici, F., Cernescu, C., Campbell, G. L. \& Nedelcu, N. I. West Nile

703 encephalitis epidemic in southeastern Romania. Lancet 352, 767-771 (1998).

704 8. Bakonyi, T. \& Haussig, J. M. West Nile virus keeps on moving up in Europe. Euro

705 Surveill 25, (2020).

706 9. Fontaine, A., Jiolle, D., Moltini-Conclois, I., Lequime, S. \& Lambrechts, L. Excretion

707 of dengue virus RNA by Aedes aegypti allows non-destructive monitoring of viral

708 dissemination in individual mosquitoes. Sci Rep 6, 24885 (2016).

709 10. Meyer, D. B., Ramirez, A. L., van den Hurk, A. F., Kurucz, N. \& Ritchie, S. A.

710 Development and Field Evaluation of a System to Collect Mosquito Excreta for the Detection

711 of Arboviruses. J Med Entomol 56, 1116-1121 (2019).

712 11. Ramírez, A. L. et al. Mosquito excreta: A sample type with many potential

713 applications for the investigation of Ross River virus and West Nile virus ecology. PLoS Negl

714 Trop Dis 12, e0006771 (2018). 
715 12. Ramesh, A. et al. Development of an urban molecular xenomonitoring system for

716 lymphatic filariasis in the Recife Metropolitan Region, Brazil. PLoS Negl Trop Dis 12,

717 e0006816 (2018).

718 13. Beck, C. et al. Contrasted Epidemiological Patterns of West Nile Virus Lineages 1 and

7192 Infections in France from 2015 to 2019. Pathogens 9, E908 (2020).

720 14. Sotelo, E. et al. Phylogenetic relationships of Western Mediterranean West Nile virus

721 strains (1996-2010) using full-length genome sequences: single or multiple introductions? J

722 Gen Virol 92, 2512-2522 (2011).

723 15. Folmer, O., Black, M., Hoeh, W., Lutz, R. \& Vrijenhoek, R. DNA primers for

724 amplification of mitochondrial cytochrome c oxidase subunit I from diverse metazoan

725 invertebrates. Mol Mar Biol Biotechnol 3, 294-299 (1994).

726 16. Callahan, B. J. et al. DADA2: High-resolution sample inference from Illumina

727 amplicon data. Nat Methods 13, 581-583 (2016).

728 17. Nagy, A. et al. Extraordinary increase in West Nile virus cases and first confirmed

729 human Usutu virus infection in Hungary, 2018. Euro Surveill 24, (2019).

730 18. Autorino, G. L. et al. West Nile virus epidemic in horses, Tuscany region, Italy.

731 Emerg Infect Dis 8, 1372-1378 (2002).

732 19. Jurado-Tarifa, E. et al. Monitoring of West Nile virus, Usutu virus and Meaban virus

733 in waterfowl used as decoys and wild raptors in southern Spain. Comp Immunol Microbiol

734 Infect Dis 49, 58-64 (2016).

735 20. Faggioni, G. et al. Prevalence of Usutu and West Nile virus antibodies in human sera,

736 Modena, Italy, 2012. J Med Virol 90, 1666-1668 (2018).

737 21. Schvartz, G. et al. Exposure of Horses in Israel to West Nile Virus and Usutu Virus.

738 Viruses 12, E1099 (2020).

739 22. Ziegler, U. et al. Epidemic Spread of Usutu Virus in Southwest Germany in 2011 to

7402013 and Monitoring of Wild Birds for Usutu and West Nile Viruses. Vector Borne Zoonotic

741 Dis 15, 481-488 (2015).

742 23. Zaaijer, H. L., Slot, E., Molier, M., Reusken, C. B. E. M. \& Koppelman, M. H. G. M.

743 Usutu virus infection in Dutch blood donors. Transfusion 59, 2931-2937 (2019).

744 24. Percivalle, E. et al. West Nile or Usutu Virus? A Three-Year Follow-Up of Humoral

745 and Cellular Response in a Group of Asymptomatic Blood Donors. Viruses 12, E157 (2020).

746 25. Calzolari, M. et al. Enhanced West Nile Virus Circulation in the Emilia-Romagna and

747 Lombardy Regions (Northern Italy) in 2018 Detected by Entomological Surveillance. Front

748 Vet Sci 7, 243 (2020).

749 26. Eidson, M. et al. Dead bird surveillance as an early warning system for West Nile

750 virus. Emerg Infect Dis 7, 631-635 (2001).

751 27. Eidson, M. et al. Dead crow density and West Nile virus monitoring, New York.

752 Emerg Infect Dis 11, 1370-1375 (2005).

753 28. Ramírez, A. L. et al. Metagenomic Analysis of the Virome of Mosquito Excreta.

754 mSphere 5, e00587-20 (2020).

755 29. Ramírez, A. L. et al. Stability of West Nile Virus (Flaviviridae: Flavivirus) RNA in

756 Mosquito Excreta. J Med Entomol 56, 1135-1138 (2019).

757 30. Ramírez, A. L. et al. Malaria surveillance from both ends: concurrent detection of

758 Plasmodium falciparum in saliva and excreta harvested from Anopheles mosquitoes. Parasit

759 Vectors 12, 355 (2019).

760 31. Pilotte, N., Zaky, W. I., Abrams, B. P., Chadee, D. D. \& Williams, S. A. A Novel

761 Xenomonitoring Technique Using Mosquito Excreta/Feces for the Detection of Filarial

762 Parasites and Malaria. PLoS Negl Trop Dis 10, e0004641 (2016).

763 32. Murgue, B. et al. West Nile outbreak in horses in southern France, 2000: the return

764 after 35 years. Emerg Infect Dis 7, 692-696 (2001). 
765 33. Rageau, J. \& Mouchet, J. Les arthropodes hématophages de Camargue. Cahier de

766 l'ORSTOM, série entomologie médicale et parasitologie 5, 261-281 (1967).

767 34. Nasci, R. S. et al. West Nile virus in overwintering Culex mosquitoes, New York City,

768 2000. Emerg Infect Dis 7, 742-744 (2001).

769 35. Rudolf, I. et al. West Nile virus in overwintering mosquitoes, central Europe.

$770 \quad$ Parasites Vectors 10, 452 (2017).

771 36. Wheeler, S. S. et al. Detection of persistent west nile virus RNA in experimentally and

772 naturally infected avian hosts. Am J Trop Med Hyg 87, 559-564 (2012).

773 37. Reisen, W. K. et al. Overwintering of West Nile virus in Southern California. J Med

774 Entomol 43, 344-355 (2006).

775 38. Hajibabaei, M., Porter, T. M., Wright, M. \& Rudar, J. COI metabarcoding primer

776 choice affects richness and recovery of indicator taxa in freshwater systems. PLoS One 14,

777 e0220953 (2019).

778 39. Mechai, S. et al. Mosquito Identification From Bulk Samples Using DNA

779 Metabarcoding: a Protocol to Support Mosquito-Borne Disease Surveillance in Canada. $J$

780 Med Entomol 58, 1686-1700 (2021).

781 40. Reeves, L. E., Gillett-Kaufman, J. L., Kawahara, A. Y. \& Kaufman, P. E. Barcoding

782 blood meals: New vertebrate-specific primer sets for assigning taxonomic identities to host

783 DNA from mosquito blood meals. PLoS Negl Trop Dis 12, e0006767 (2018).

784 41. Salter, S. J. et al. Reagent and laboratory contamination can critically impact

785 sequence-based microbiome analyses. BMC Biol 12, 87 (2014).

786 42. Osório, H. C., Zé-Zé, L., Amaro, F., Nunes, A. \& Alves, M. J. Sympatric occurrence

787 of Culex pipiens (Diptera, Culicidae) biotypes pipiens, molestus and their hybrids in Portugal,

788 Western Europe: feeding patterns and habitat determinants. Med Vet Entomol 28, 103-109

789 (2014).

790 43. Yurchenko, A. A. et al. Genomic differentiation and intercontinental population

791 structure of mosquito vectors Culex pipiens pipiens and Culex pipiens molestus. Sci Rep 10,

$7927504(2020)$.

793 44. M Hernández-Triana, L. et al. DNA barcoding of British mosquitoes (Diptera,

794 Culicidae) to support species identification, discovery of cryptic genetic diversity and

795 monitoring invasive species. Zookeys 832, 57-76 (2019).

796 45. Gunay, F., Alten, B., Simsek, F., Aldemir, A. \& Linton, Y.-M. Barcoding Turkish

797 Culex mosquitoes to facilitate arbovirus vector incrimination studies reveals hidden diversity

798 and new potential vectors. Acta Trop 143, 112-120 (2015).

799 46. Taieb, L. et al. Bird Species Involved in West Nile Virus Epidemiological Cycle in

800 Southern Québec. Int J Environ Res Public Health 17, E4517 (2020).

801 47. Grubaugh, N. D. et al. Xenosurveillance: a novel mosquito-based approach for

802 examining the human-pathogen landscape. PLoS Negl Trop Dis 9, e0003628 (2015).

803 48. Jourdain, E. et al. Bird species potentially involved in introduction, amplification, and

804 spread of West Nile virus in a Mediterranean wetland, the Camargue (Southern France).

805 Vector Borne Zoonotic Dis 7, 15-33 (2007).

806 49. Buckley, A. et al. Serological evidence of West Nile virus, Usutu virus and Sindbis

807 virus infection of birds in the UK. J Gen Virol 84, 2807-2817 (2003).

808 50. Jourdain, E. et al. West Nile virus in wild resident birds, Southern France, 2004.

809 Vector Borne Zoonotic Dis 7, 448-452 (2007).

810 51. Ezenwa, V. O., Godsey, M. S., King, R. J. \& Guptill, S. C. Avian diversity and West

811 Nile virus: testing associations between biodiversity and infectious disease risk. Proc Biol Sci

812 273, 109-117 (2006).

813 52. Dobson, A. Population dynamics of pathogens with multiple host species. Am Nat 164

814 Suppl 5, S64-78 (2004). 
815 53. Timmins, D. R. et al. Modifying the Biogents Sentinel Trap to Increase the Longevity 816 of Captured Aedes aegypti. J Med Entomol 55, 1638-1641 (2018).

817 54. Bahuon, C. et al. West Nile virus epizootics in the Camargue (France) in 2015 and

818 reinforcement of surveillance and control networks. Rev Sci Tech 35, 811-824 (2016).

819 55. Murgue, B., Murri, S., Triki, H., Deubel, V. \& Zeller, H. G. West Nile in the

820 Mediterranean basin: 1950-2000. Ann N Y Acad Sci 951, 117-126 (2001).

821

822

823

824

825

826

827

828

829 56. Holleley, C. \& Sutcliffe, A. 96 Well DNA Extraction Protocol. (MR4, 2009).

57. Quick, J. et al. Multiplex PCR method for MinION and Illumina sequencing of Zika and other virus genomes directly from clinical samples. Nat Protoc 12, 1261-1276 (2017).

58. Grubaugh, N. D. et al. An amplicon-based sequencing framework for accurately measuring intrahost virus diversity using PrimalSeq and iVar. Genome Biol 20, 8 (2019).

59. Bolger, A. M., Lohse, M. \& Usadel, B. Trimmomatic: a flexible trimmer for Illumina sequence data. Bioinformatics 30, 2114-2120 (2014).

60. Langmead, B. \& Salzberg, S. L. Fast gapped-read alignment with Bowtie 2. Nat Methods 9, 357-359 (2012).

830 61. Li, H. et al. The Sequence Alignment/Map format and SAMtools. Bioinformatics 25, $8312078-2079$ (2009).

832 62. Quinlan, A. R. \& Hall, I. M. BEDTools: a flexible suite of utilities for comparing

833 genomic features. Bioinformatics 26, 841-842 (2010).

834 63. Gouy, M., Tannier, E., Comte, N. \& Parsons, D. P. Seaview Version 5: A

835 Multiplatform Software for Multiple Sequence Alignment, Molecular Phylogenetic Analyses,

836 and Tree Reconciliation. Methods Mol Biol 2231, 241-260 (2021).

837 64. Lefort, V., Longueville, J.-E. \& Gascuel, O. SMS: Smart Model Selection in PhyML.

838 Mol Biol Evol 34, 2422-2424 (2017).

839 65. Yu, G. Using ggtree to Visualize Data on Tree-Like Structures. Curr Protoc

840 Bioinformatics 69, e96 (2020).

$841 \quad 66 . \quad$ Leigh, J. W. \& Bryant, D. Full $\square$ feature software for haplotype network construction.

842 Methods Ecol Evol 6, 1110-1116 (2015).

843 67. Clement, M., Posada, D. \& Crandall, K. A. TCS: a computer program to estimate gene 844 genealogies. Mol Ecol 9, 1657-1659 (2000).

845 68. Elbrecht, V. \& Leese, F. Can DNA-Based Ecosystem Assessments Quantify Species

846 Abundance? Testing Primer Bias and Biomass--Sequence Relationships with an Innovative

847 Metabarcoding Protocol. PLoS One 10, e0130324 (2015).

848 69. O'Rourke, D. Building a COI database from BOLD references.

849 https://forum.qiime2.org/t/building-a-coi-database-from-bold-references/16129.

850 70. Kassambara, A. ggpubr: 'ggplot2' Based Publication Ready Plots. (2020).

851 71. Bates, D., Mächler, M., Bolker, B. \& Walker, S. Fitting Linear Mixed-Effects Models

852 Using lme4. J. Stat. Soft. 67, (2015).

853 72. Nguyen, L.-T., Schmidt, H. A., von Haeseler, A. \& Minh, B. Q. IQ-TREE: a fast and

854 effective stochastic algorithm for estimating maximum-likelihood phylogenies. Mol Biol Evol 855 32, 268-274 (2015).

856 73. Fernandes, A. D. et al. Unifying the analysis of high-throughput sequencing datasets:

857 characterizing RNA-seq, 16S rRNA gene sequencing and selective growth experiments by

858 compositional data analysis. Microbiome 2, 15 (2014).

859 74. McMurdie, P. J. \& Holmes, S. phyloseq: an R package for reproducible interactive

860 analysis and graphics of microbiome census data. PLoS One 8, e61217 (2013).

861 75. Wickham, H. ggplot2: Elegant Graphics for Data Analysis. (Springer International

862 Publishing $\square$ : Imprint: Springer, 2016). doi:10.1007/978-3-319-24277-4.

863 76. Wickham, H. et al. Welcome to the Tidyverse. JOSS 4, 1686 (2019). 
A

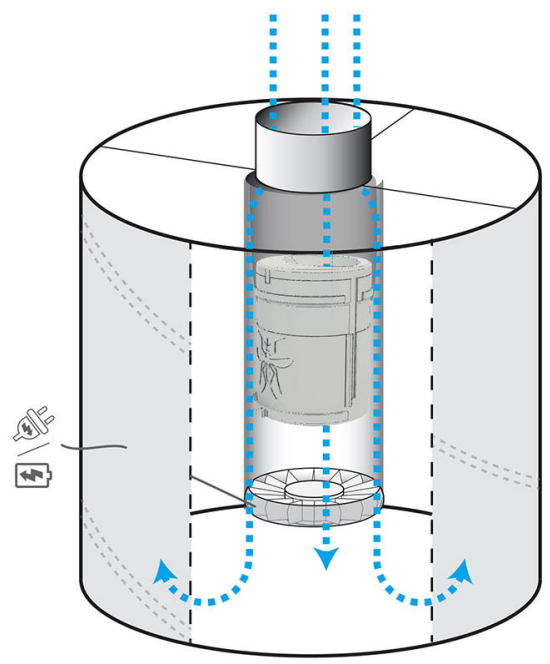

B

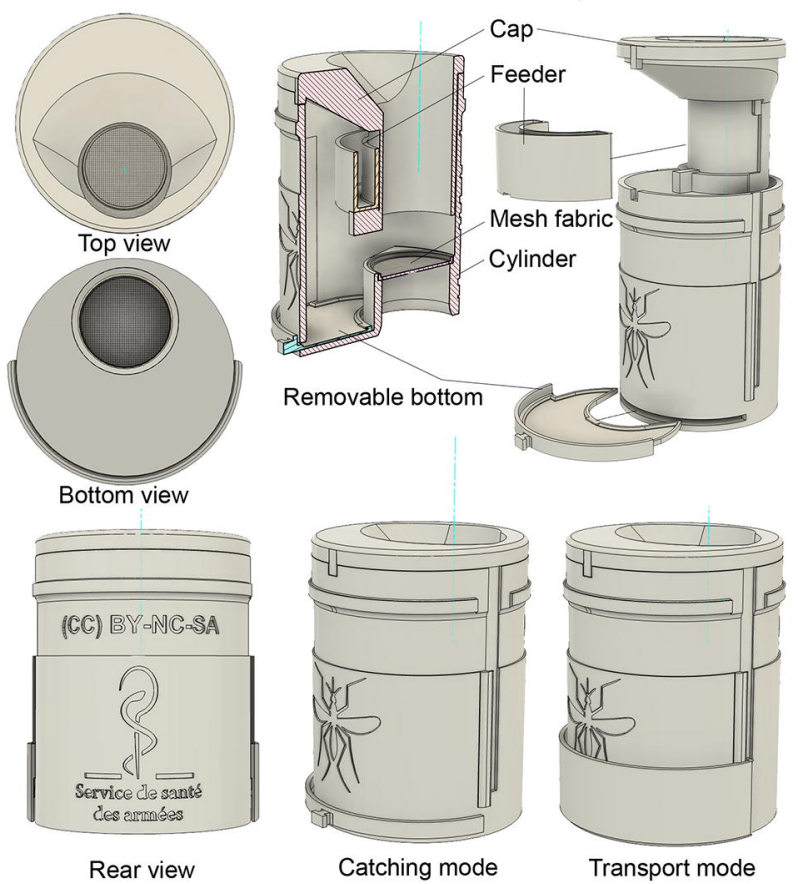




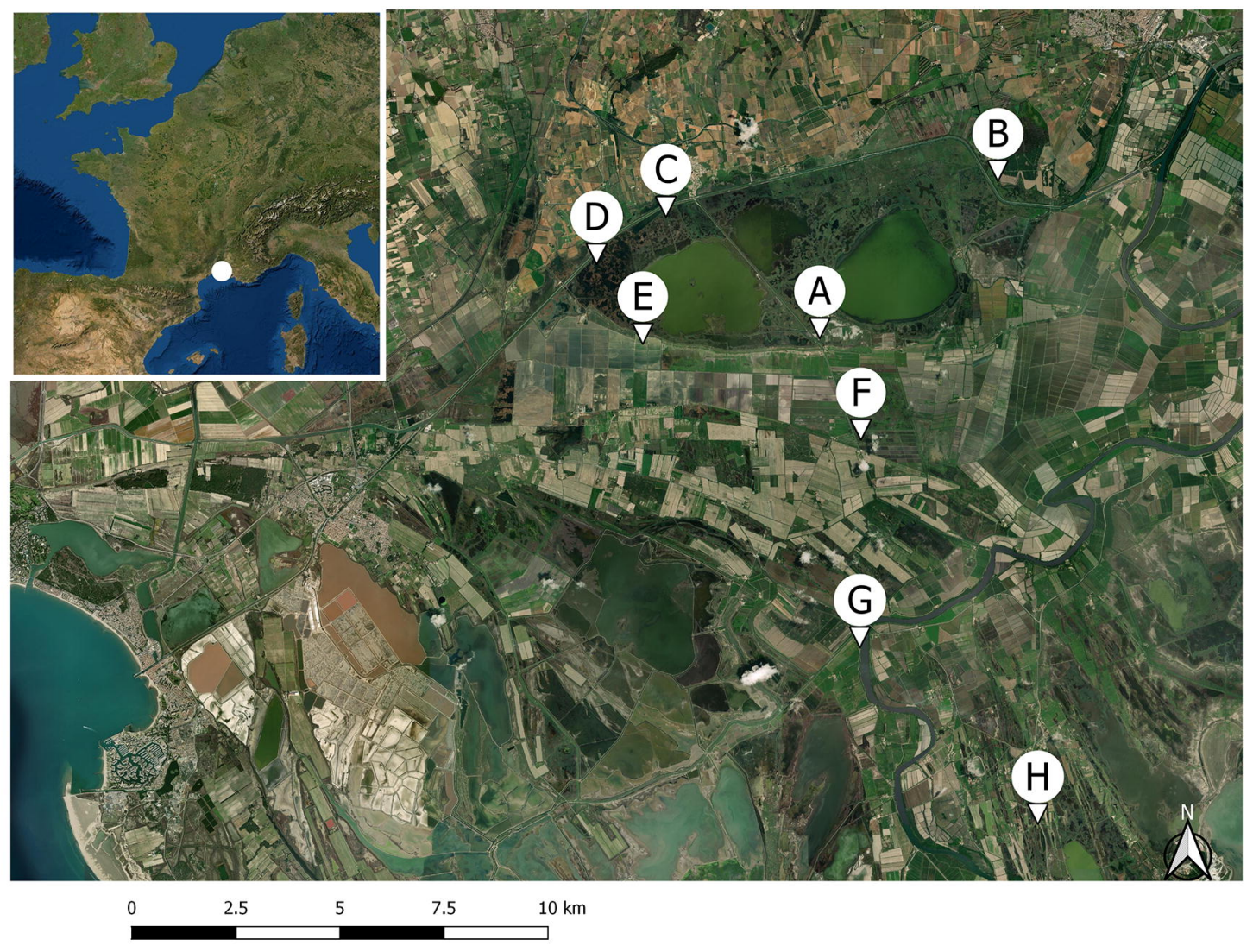




\section{Spain 2007 golden eagle \\ Romania/Russia} 1996-2000

Human/Culex pipiens (AF260969, AY278442,

\section{AF317203, AY277252)}

Italy 2011 Human (JQ928174)

Italy 2011/2013 Human (JQ928174, KF647253)
(FJ766332, FJ766331) Italy 1998 horse (AF404757)

Italy 2011 Human (JN858069, JQ928175)

Morocco 2003 horse

(AY701413)

France 2004 magpie (DQ786573, DQ786572)

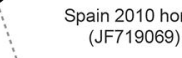

France 1996/2000 horse (AY268132, AY701412)

France 2015 horse (MT863559)

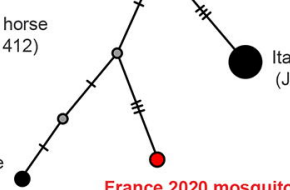

France 2020 mosquito excreta (F-15-09)

\section{1}

97
GU011992_Italy_2009_Human

72 KF234080_Italy_2009_Human

JF719065_Italy_2008_jay

JF719066_Italy_2008_magpie

-JF719069_Spain_2010_horse

61

-F-15-09_2020_France_mosquito excreta

- MT863559_France_2015 horse

,DQ786572_France_2004_house sparrow

DQ786573_France_2004_magpie

AY701413_Morocco_2003_horse

IFJ766331_Spain_2007_golden eagle

FJ766332_Spain_2007_golden eagle

-AF404757 Italy 1998 horse

J JQ928175_Italy_2011_Human

JN858069_Italy_2011_Human

JQ928174_Italy_2011_Human

KF647253_Italy_2013_Human

- AY268132_France_2000_horse

100

AY701412_Morocco_1996_horse

|AY277252_Russia_1999_Human

AF317203_Russia_1999_Human

AY278442_Russia_2000_Human

100

AF260969_Romania_1996_Culex pipiens

NC 009942 Refseq lineage 1

D00246_Kunjin_virus_Australia_1960_Mosquito DQ256376_India_1980_Human

MT863560_France_2018_Common_buzzard

MT863561_France_2018_Goshawk

KP789958_Italy_2014_Human

KF647251_Italy_2013_Human

MF984344_Austria_2015_goshawk

MW142223_Germany_2020_Human

MF984349_Austria_2016_horse

DQ116961_Hungary_2005_goshawk

-NC_001563_Refseq_lineage2

-AY765264_Czech_Rep._1997_Culex 


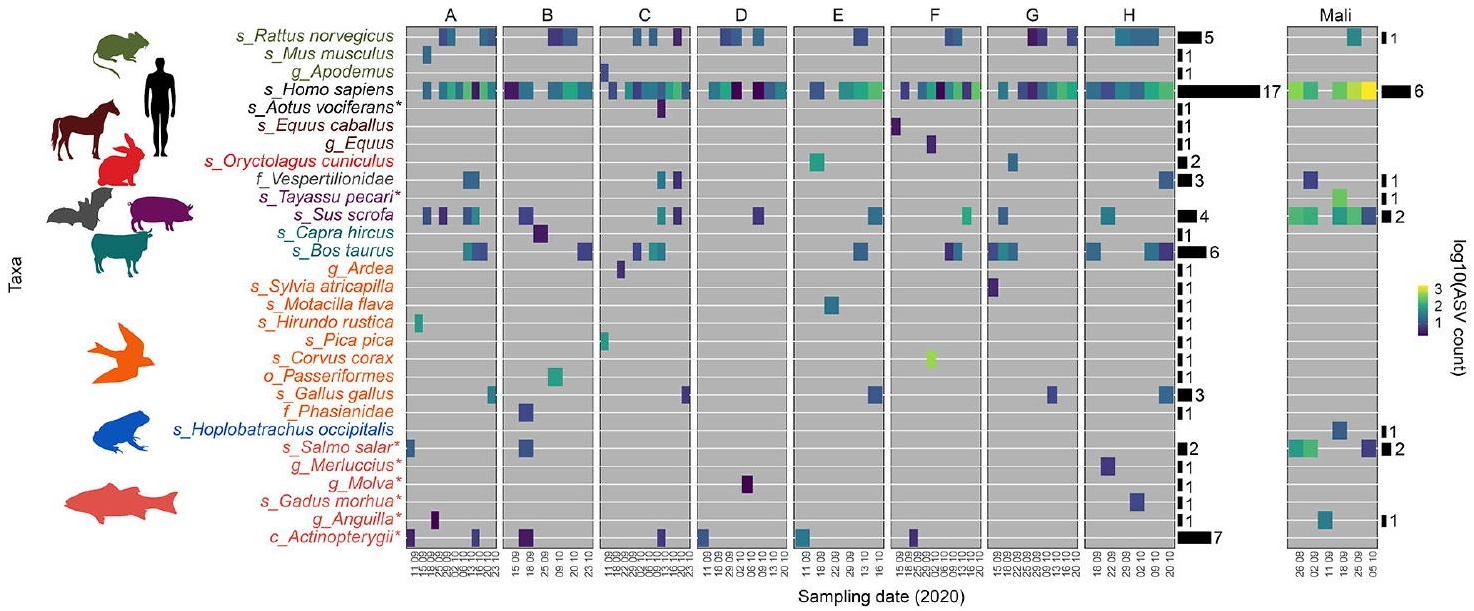

